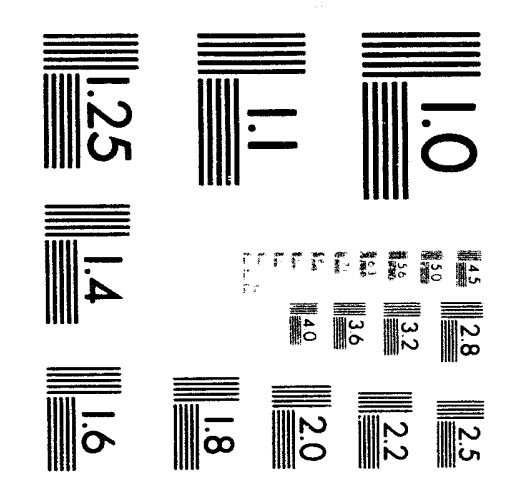



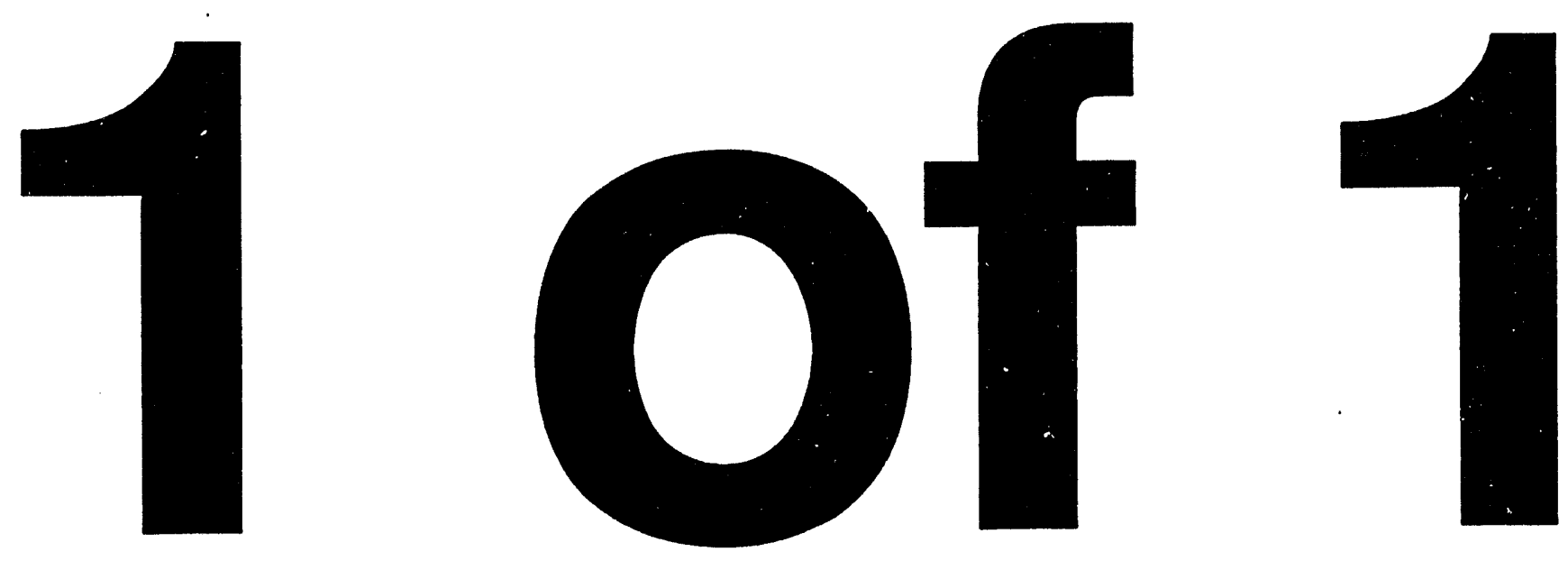


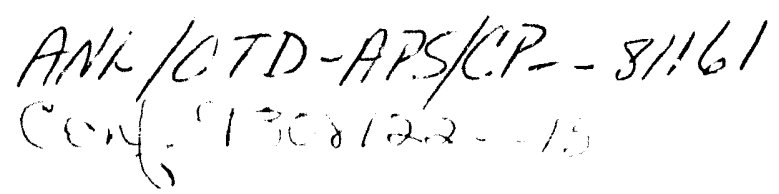

\section{Third Generation Synchrotron Radiation Applied to Materials Science}

Elton N. Kaufmann, Office of the Director and Materials Science Division Wenbing Yun, Experimental Facilities Division, Advanced Photon Source Argonne National Laboratory, Argonne, Illinois 60439, U.S.A.

The utility of synchrotron radiation for the characterization of materials and the ramifications of the availability of the new thind-generation, high-energy, high-intensity sources of synchrotron radiation are discussed. Some specific examples are given of the power of $\mathrm{x}$-ray analysis techniques to be expected with these new machines.

\section{INTRODUC'TION}

X-rays have a long and productive history in the field of materials characterization, and in more recent times, in their use for the modification of materials. The character of the problems studied has become more sophisticated and detection sensitivity and spatial resolution have continually improved to the point where even the most intense laboratory $x$-ray sources, such as rotating anode sources, are inadequate to many tasks. Well over two decades ago, researchers began to use synchrotron radiation provided parasitically at existing charged particle accelerators devoted primarily to nuclear and high energy physics. As the utility of synchrotron radiation became more widely appreciated and demand increased, parasitic operation could no longer support the community. Dedicated facilities were therefore constructed and have been generically referred to as "second-generation" synchrotron radiation facilities. Demand for this type of facility has once again exceeded supply and, over the intervening years, technology has advanced to the point where machines of much greater capability can and are being built.

The latter therefore are referred to as "third-generation" machines. Although several machines are designed to provide radiation in the soft $x$-ray and vacuum ultraviolet spectral regions, the purpose of this paper is to summarize some of the benefits specifically associated with new machines capable of significant intensity in the hard $x$-ray region $(>\sim 3 \mathrm{keV})$. The wide variety of synchrotron radiation tools and techniques already in use is described in several conference proceedings.[1-3]

The unprecedented high brilliance and high coherent power that will be provided by the third-generation synchrotron $x$-ray sources will open up new opportunities in imaging and interferometry. The high brilliance $\mathrm{x}$-ray beams can bring a large flux of $x$-ray photons to a small focal point. Therefore, powerful $x$-ray microscopic probes with submicron spatial resolution can be constructed and spatially-resolved $x$-ray microspectroscopy, microdiffraction, microanalysis of elements, and threedimensional microimaging can be developed. The high coherent power may also be used to extend many coherence-based techniques developed in the visible part of the electromagnetic spectrum to the $x$-ray spectral region.

At present there are three high-energy third-generation machines either in operation or under construction. The first is the European Synchrotron Radiation Facility (ESRF) in Grenoble, France, which has been operating for about one year at this writing. The two facilities in the design and construction phase are the Advanced Photon Source (APS) at the Argonne National Laboratory (Argonne, USA) due to operate in late 1995 and the Spring- 8 facility at Harima Science Garden City in Japan due to operate in 1998. For ESRF, APS, and Spring- 8 , the design energies for the beam of 


\section{DISCLAIMER}

This report was prepared as an account of work sponsored by an agency of the United States Government. Neither the United States Government nor any agency thereof, nor any of their employees, makes any warranty, express or implied, or assumes any legal liability or responsibility for the accuracy, completeness, or usefulness of any information, apparatus, product, or process disclosed, or represents that its use would not infringe privately owned rights. Reference herein to any specific commercial product, process, or service by trade name, trademark, manufacturer, or otherwise does not necessarily constitute or imply its endorsement, recommendation, or favoring by the United States Government or any agency thereof. The views and opinions of authors expressed herein do not necessarily state or reflect those of the United States Government or any agency thereof. 


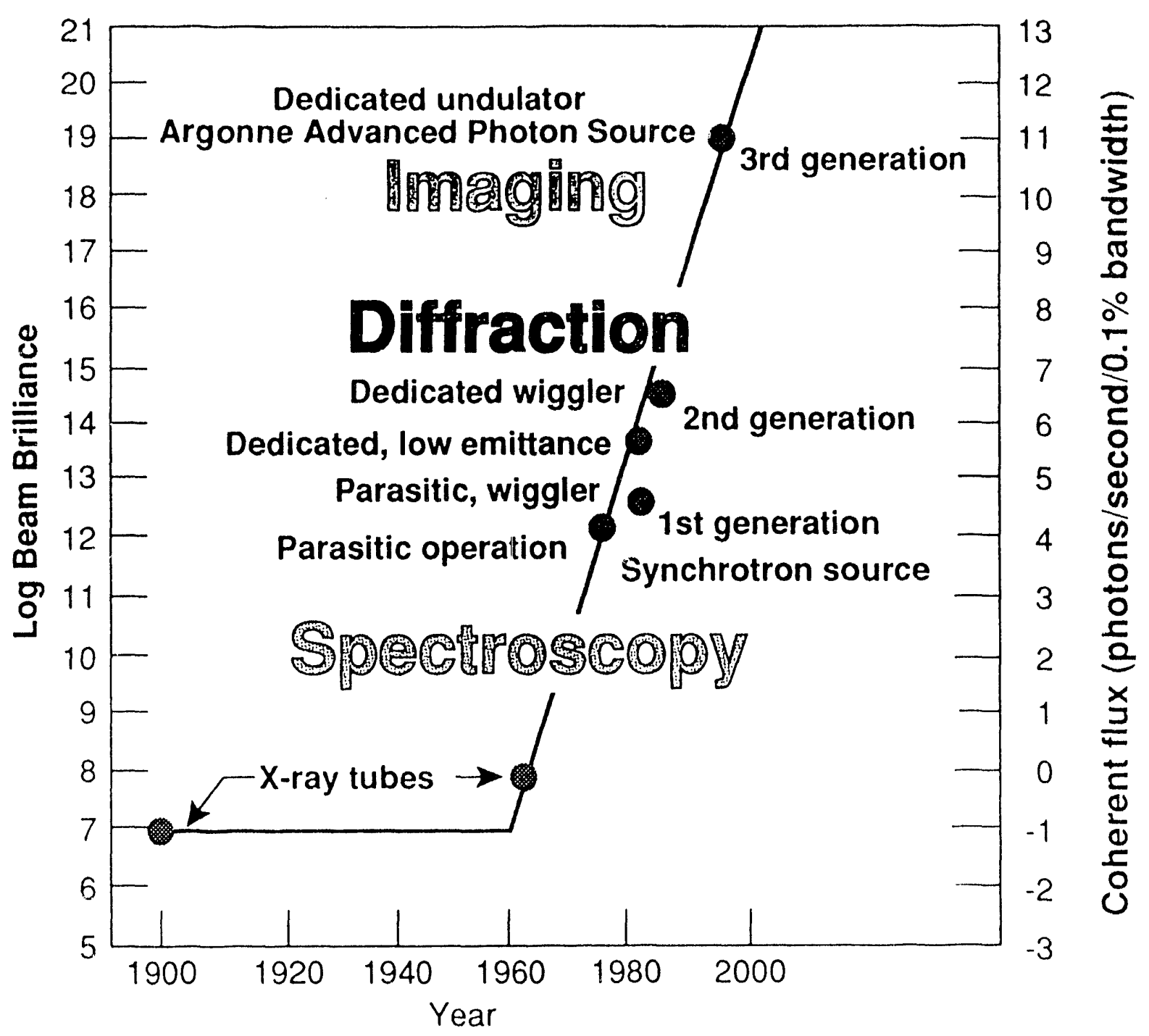

Fig. 1 History of 8-keV x-ray sources. Time-average spectral brilliance and coherent flux calculated for the APS and other $x$-ray sources.

positrons or electrons in the storage ring are respectively 6,7 , and $8 \mathrm{GeV}$.

At these particle energies, the intensity of continuous spectrum Bremsstrahlung reaches its maximum at higher photon energies, well into and beyond the soft $\mathrm{x}$-ray region. It is the improved designs and greater stability of these machines that allow substantially greater brilliance. The most notable advances in photon production are the "wigglers" and "undulators," collectively known as insertion devices.
These add an oscillatory acceleration to the particle beam that provides yet higher, tunable $\mathrm{x}$-ray energies and improved collimation, polarization and coherence characteristics.

Intrinsic to the operation of the synchrotron, because the charged particle beam in the ring is bunched, is the time structure of the x-ray source. Advantage can be taken of the pulsed nature of the source in doing time-dependent characterization. The new machines will allow both extension 
of methods presently applied today as well as new methods which heretofore were not feasible.

To illustrate the power of the new $x$-ray sources, we will discuss a few particular examples.

\section{APPLICATIONS USING COHERENCE}

A radiation source with adequate coherent power offers the unique capability of studying both the amplitude and the phase distributions of a signal wave by detecting its interference with another mutually coherent wave, whose wavefront is known.[4] techniques based on this property have made a significant impact on science and technology as well as on our daily lives with the invention of the optical laser and the development of other coherent optics. The coherent $x$-ray flux emitted from the undulator of a third-generation $x$-ray source, such as APS, at a wavelength of one Angstrom and a spectral purity $\lambda / \Delta \lambda=1000$ is expected to exceed $10^{9}$ photons/sec. This is several orders of magnitude greater than that from the most brilliant $x$-ray sources available today (see Fig. 1).[5] The availability of such high power coherent $x$-ray sources opens up excellent opportunities for extending the coherence-based techniques developed in the visible part of the electromagnetic spectrum to the $\mathrm{x}$-ray spectral regime.

A coherence-based signal enhancement technique is well developed in the visible light region and can be applied to the $x$-ray region. The technique relies on the changes of the interference intensity of two or more mutually coherent beams depending on the relative phase of the two beams at the point of detection (provided that the polarization of the two beams is the same). By adjusting the relative phase of two such interfering beams, one being a signal wave to be detected and the other a reference wave, the strength of the signal is either enhanced or suppressed. The enhancement or suppression factor can be many orders of magnitude. The dependence of interference on polarization may also be used to enhance $\mathrm{cr}$ suppress certain polarization components of the signal by adjusting the polarization of the reference beam. For example, detection of $x$-rays scattered by the magnetic moment of a system may be enhanced by several orders of magnitude over nonmagnetic scattering. Such an enhancement could greatly increase the usefulness of magnetic $x$-ray scattering for materials research.

One particular characterization method that depends on the coherence of the beam is $\mathrm{x}$-ray speckle spectroscopy. It is also known as dynamic light scattering, intensity fluctuation spectroscopy, or light-beating spectroscopy. It has been widely used with visible light to study processes such as critical fluctuations near phase transitions in fluids and the diffusion of particles in liquids.[6,7] The length scale accessible to study may be reduced by several orders of magnitude if this technique is extended into the $x$-ray spectral region. Recently, x-ray speckle patterns have been observed using the second-generation $x$-ray sources in the Bragg-diffracted beam from a single crystal of binary alloy $\mathrm{Cu}_{3} \mathrm{Au}$ with randomly arranged antiphase domains[8] and from gold-coated films of symmetric diblock copolymers of polystyrene (PS) and poplymethylmethcrylate (PMMA).[9] Those successful demonstrations open many exciting possibilities to study nonequilibrium and equilibrium systems on length scales of $1-100 \mathrm{~nm}$ and $1-10,000 \mathrm{~nm}$ for volumetric and surface studies, respectively. -

The primary requirement for $x$-ray speckle spectroscopy is an illumination beam of adequate spatial and temporal coherence. That is achieved through use of a monochromator and a small aperture. This coherent flux and the detection efficiency of a position sensitive detector determine the time resolution achievable for study of dynamic and kinetic processes. Opportunities for studying dynamics with ever improving time resolution will result from increased $x$-ray coherent flux and advances in coherent $x$-ray optics and $x$-ray detectors. 
Many types of $x$-ray interferometric techniques have been developed since the successful demonstration of an $x$-ray interferometer by Bonse and Hart.[10] This has allowed use of the relative phase difference between two or more interfering $x$-ray beams. Many unique capabilities have been shown including the study of defects in nearly perfect crystals, the precise measurement of atomic scattering factors, the absolute measurement of lattice parameters, and the precise measurement of small displacements and fundamental constants. Recently, an x-ray Michelson interferometer able to continuously scan the phase difference was constructed.[11] However, the energy bandwidth is restricted and the relative phase change is complicated by defocusing and the use of Bragg diffraction. Several different approaches to the development of $\mathbf{x}$-ray Michelson interferometers are been considered.[12,13] Some novel applications being considered includes high resolution Fourier transform $x$-ray spectroscopy and Fourier transform $x$-ray imaging of surfaces and interfaces.

\section{IMAGING}

As indicated earlier, the high brilliance enables focusing a large flux of $x$-ray photons at a focal point. X-ray wavelengths about three orders of magnitude shorter than that of visible light permit spatial resolution far better than obtainable in the visible. The combination of the small focal spot, the moderate $x$-ray interaction cross section with matter, the contrast available from use of absorption edges, and elemental sensitivity (e.g., by detecting characteristic fluorescence $\mathrm{x}$-rays) provide excellent opportunities to image and probe for structural and materials analyses. Specifically, these probes can be used to map elemental distributions by $\mathrm{x}$-ray fluorescence or absorption or to map phase and strain distributions in a material using $x$-ray microdiffraction.

Rapid advances in the last decade in microfabrication technology, high precision surface finishing, crystal growth, controlled

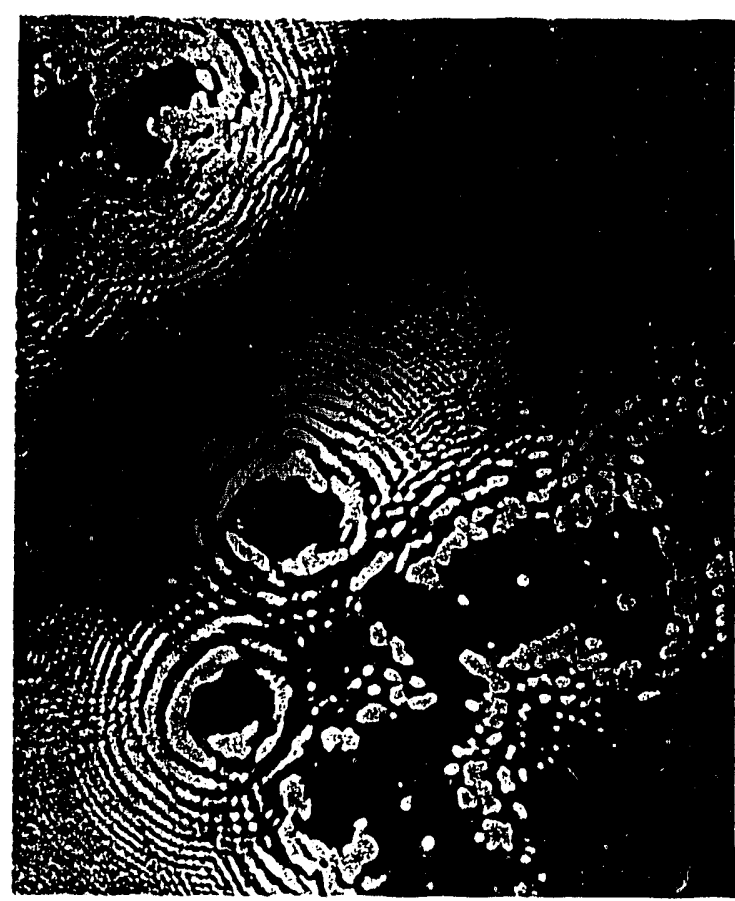

Fig. 2 Transmission electron micrograph of several zymogen granules obtained with soft $\mathrm{x}$-ray photons using Gabor geometry (from Ref. 19).

layer deposition, precision metrology, and high quality position sensitive $\mathrm{x}$-ray detectors account for great strides in the fabrication of high-spatial resolution $\mathrm{x}$-ray microfocusing optics.[14] These advances have allowed for the extraordinarily rapid development in $x$-ray microfocusing-based techniques. A Fresnel zone plate with $300 \mathrm{~nm}$ resolution for soft $x$-ray application has been constructed.[15] Soft x-ray holography, micro-imaging, micro-analysis, and microspectroscopy have been developed with spatial resolution far better than that obtainable with a visible light microscope. $[16,17,18]$ High quality soft $x$-ray holograms have also recently been obtained with a soft x-ray undulator source. [19,20] Fig. 2 shows a transmission electron micrograph of an $x$-ray hologram of several zymogen granules obtained using the Gabor (in line) geometry. Several types of optical elements capable of focusing $x$-rays to a submicron spot for $\mathrm{x}$-ray energies greater 
than $5 \mathrm{keV}$ have also been developed.[21-24] New capabilities for microanalysis, microspectroscopy, and microdiffraction have been demonstrated with those focusing optics. For example, spatially-resolved $x$-ray microdiffraction was used for mapping crystallite orientation in a photographic film.[25] X-ray microspectroscopy from an area illuminated by a submicron focused beam was also demonstrated.[21,25]

The availability of the third-generation $x$-ray sources will allow many conventional $\mathrm{x}$-ray techniques to be applied to samples of small linear dimension. For example, it is expected that $\mathrm{x}$-ray microanalysis with submicron spatial resolution and femtogram sensitivity can be obtained.[26] Materials not available in larger quantities can thus be analyzed. In addition, x-ray phase contrast microscopy, similar to that in the visible light regime, can be developed with the high coherent $x$-ray flux as has been demonstrated in the soft $x$-ray spectral region using an appropriate phase shifter.[27] In the $x$-ray spectral region, the real part of the atomic scattering factor is generally larger than its imaginary counterpart. As a consequence, phase contrast arising from elemental composition and spatial distribution in a sample is better for imaging than absorption-based contrast. This phase contrast can be further enhanced by interfering with a coherent reference beam. The increase in contrast not only increases the signal-to-noise ratio, but simultaneously reduces the minimum necessary total fluence of $x$-rays on the sample. Radiation damage, which is of great concern in biological samples, can thus be reduced.[28]

\section{SURFACE AND INTERFACES}

The high degree of angular collimation and high brilliance of synchrotron radiation compared with conventional $x$-ray sources has been particularly important for the development of various surface $x$-ray techniques in the last two decades. The surface $x$-ray techniques include $x$-ray

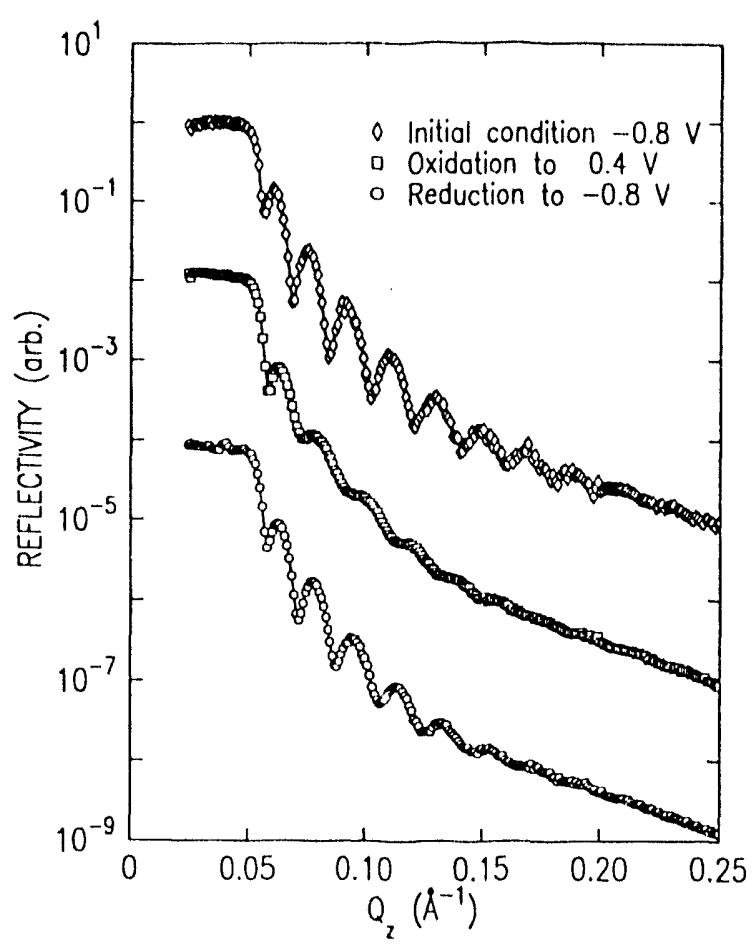

Fig. 3 X.ray reflectivity measured from a $\mathrm{Cu}$-on-Si electrode at various potentials in borate buffer solution ( $\mathrm{pH}$ 8.4) (From Ref. 31).

specular reflectance (SF), grazing incidence diffraction (GID), near total fluorescence (NTF), surface standing waves (SSW), surface speckle (SS), surface spectroscopy (SSP), and others. The surface sensitivity of these techniques relies on the steep drop in $x$-ray intensity inside the condensed material along the surface normal when the incidence angle is smaller than the critical angle for total reflection.

The surface techniques have been applied recently to a broad range of research For example, $x$-ray reflectivity measurement has been applied to liquid-vapor interfaces, microemulsion surfaces, and to the in-situ study of electrochemical processes at solution-electrode interfaces.[29-31] Fig. 3 shows $\mathrm{x}$-ray reflectance from a Cu-on-Si electrode at various potentials in a borate buffer solution (pH 8.4).[31] The oscillation in the reflectance is related to the thickness and roughness of the oxide film formed at 
the $\mathrm{Cu}$-electrolyte interface.

The near total external fluorescence technique and surface $x$-ray spectroscopy have been used to investigate the adsorption of a monolayer of counterions at the water-air interface.[32] The grazing incidence $x$-ray method has been used to study melting of Pd monolayers on $\mathrm{Cu}(110)$ surfaces and atomic structure at electrode interfaces.[33,34] X-ray standing wave studies of oxidation at a $\mathrm{Cu}$ electrode in electrolyte has also been reported.[35] And, the distribution of crystallographic structure along the surface normal of iron oxide thin films has been profiled using grazing incidence $x$-ray techniques.[36]

Surface $x$-ray techniques will clearly benefit from most new capabilities of the third-generation synchrotron $x$-ray sources -principally from the brilliance which leads to significant improvement in signal-to-noise ratios. Spatial and time resolution improvements coupled to selective contrast based on coherence and polarization effects will also frequently come into play.

\section{NEW TECHNOLOGICAL CAPABILITIES USING X-RAYS}

While the advances in microfabrication and precise metrology have been essential to the development of much innovative x-ray instrumentation, substantial new technological capabilities have also derived from this development. For example, synchrotronbased $x$-ray deep lithography has fabricated micromechanical components that have applications in microsensors and microactuators.[37] In fact, a fully functional planar, magnetic micromotor has been fabricated and tested.[37] Microstructures with reliefs to $500 \mu \mathrm{m}$ and nearly perfect edge acuity can be fabricated. Such thick microstructures with submicron features have also been developed.[23] The latter technique has been used to produce phase zone plates for $\mathrm{x}$-ray focusing applications. A phase zone plate with minimum line width of $0.5 \mu \mathrm{m}$ and thickness of $3.1 \mu \mathrm{m}$ has been fabricated and tested. This produced an experimentally measured x-ray focusing efficiency as high as 33\%.[23]

High brilliance of the third-generation $x$-ray sources will not only enhance the microfabrication techniques described above but will also very likely create qualitatively new capabilities for material processing and manufacturing, such as microwelding and microinspection.

\section{OTHER EXTENDED CAPABILITIES}

We can mention additional consequences of the improved $x$-ray source performance. Higher x-ray energy immodiately gains access to thicker sample materials and materials with higher $\mathrm{x}$-ray absorption cross sections, i.e., higher atomic number elements. Thus any of the x-ray characterization techniques can be extended to thicker and heavier element targets. Most particularly, imaging analysis techniques such as tomography will access larger sample sizes. Also, samples in extreme environments such as inside pressure cells, or electrode surfaces under electrolytes can be reached more easily with the greater available penetrating power.

As mentioned earlier for coherence-based techniques, phenomena which are transient or dynamic by nature can be studied with greater facility when more photons can be put on the target in a short time. Simple diffraction and imaging can be performed to study effects such as phase transitions, shock wave propagation, combustion synthesis, chemical reactions, diffusion, and even excited atomic state populations on shorter time scales with higher flux. An equally exciting consequence of higher brightness is accessibility of weak cross section interactions such as the magnetic scattering alluded to earlier. Weak signatures due to small volume fractions of the interesting phenomenon (e.g., defects, etc.) will also now be far more accessible.

In the context of these improved beam properties, the ability to tune the energy of the beam will make spectroscopy much more 
powerful. Energy selection has of course been a feature of standard $x$-ray sources through choice of voltage (for the continuous component) and anode element (for the discreet lines). With undulator radiation, however, one enjoys a spectral distribution of radiation concentrated in particular harmonics which can be adjusted continuously in energy by varying the geometry of the magnets in the undulator structure. Thus, the combination of high intensity, high signal-to-noise ratio and tunability will be the rule. The ability to adjust $x$-ray energies to correspond with elemental absorption edges for example will enhance the contrast of elemental imaging. High intensity beams of precisely determined energy will extend traditional techniques, such as photoelectron spectroscopy, to thinner surface layers with greater statistical accuracy.

In addition, the extension of Raman scattering, traditionally viewed as an optical technique, into the $\mathrm{x}$-ray region will mean lattice dynamics and molecular binding studies can be extended into previously inaccessible regions of a material sample. Such techniques will lead to mapping of not only elements but of their chemical compounds throughout a sample. Compton scattering done with greater precision and grea ter energy tunability can help determine electron momentum distributions within materials to better understand their binding and band structures.

Not only will microdiffraction, or microcrystallography, determine long-range order in small regions, but also the local structure around atomic positions, using techniques such as extended $x$-ray absorption fine structure (EXAFS) or $x$-ray absorption near edge spectroscopy (XANES), will be studied with greater statistical accuracy. More precision in the deconvolution of such spectra to yield more accurate nearest neighbor distances and positions will result. The surface analog of EXAFS will also be practical in many more cases. Finally, small angle x-ray scattering (SAXS) will be more powerful with greater statistical accuracy at higher energy, being able to "see" a wider range of extended defects, large molecules, strain fields, interfaces, and the like. We have certainly not mentioned all possibilities in this short summary. Of course, a strong role for beams of electrons and neutrons continues for many materials characterization problems ..- in some cases they are more appropriate than $\mathrm{x}$-rays.

But, the challenge for users of the third-generation synchrotron radiation is to devise the tools to control and take maximum advantage of the vastly improved properties of the available photons. In this context, we are looking at the development of more robust optical devices including lenses, monochromators, mirrors, phase shifters and focusing optics, such as Fresnel zone plates; the development of data collection methods which have the speed necessary to acquire data at the rates which will be available; developing means to handle the high heat loads which intense $x$-ray beams will entail; and, to design new applications in $\mathrm{x}$-ray materials modification and micromachining of materials.

\section{Acknowledgments}

The authors would like to thank $P$. Montano for advice in preparing this report and we are grateful to those who performed the original research which we have used as examples of synchrotron research. This work was partially supported by the U.S. Department of Energy, Office of Basic Energy Sciences, under contract W-31-109-Eng-38.

\section{References}

1. "Synchrotron Radiation in Materials Research," ed. by R. Clarke et al., Mater. Res. Soc. Proc., 143 (1989).

2. "Applications of Synchrotron Radiation Techniques to Materials Science," ed. by D.L. Perry et al., Mater. Res. Soc. Proc., 307 (1993).

3. Proceedings of the 4th International Conference on Synchrotron Radiation Instrumentation, Rev. Sci. Instrum., 63, 283-1629 (1992). 
4. Yu. I. Ostrovsky, M. M. Butusov, G. V. Ostrovskaya, Interferometry by Holography, Springer-Verlag, 1980; D. Casasent, Optical Data Processing, Springer-Verlag, 1978.

5. G.K. Shenoy, P.J. Viccaro, and D.M. Mills, Characteristics of the 7-GeV Advanced Photon Source: A Guide for Users, Argonne National Laboratory Report ANL-88-9, (1988); G.K. Shenoy and D.E. Moncton, Nucl. Instr. Meth. A266, 38 (1988).

6. P. N. Pusey, in Photon Correlation Spectroscopy and Velocimetry (H. Z. Cummins and E. P. Pike, eds.) 45-141 (Plenum, New York, 1977).

7. E. Jakeman, P. N. Pusey, and J. M. Vangnan, Opt. Commun. 17, 305-306 (1976).

8. M. Sutton, S. G. J. Mochrie, T. Greytak, S. E. Nagler, L. E. Berman, G. A. Held, and G. B. Stephenson, Nature 352, 608 (1991).

9. Z. Cai, B. Lai, W. Yun, I. McNulty, K. G. Huang, and T. P. Russell, preprint (submitted).

10. U. Bonse and M. Hart, Appl. Phys. Letters 6, 155 (1965), and 7, 99 (1965)

11. A. Appel and U. Bonse, Phys. Rev. Lett. 67, 1673 (1991).

12. M. Howells, private communication.

13. W. Yun, Z. Cai, B. Lai, and E. Gluskin, private communication.

14. N.M. Ceglio, J. X-Ray Sci. Techol. 1, 7 (1989).

15. E.H. Anderson and D. Kern, in X-Ray Microscopy III, A.G. Michette, G.R. Morrison, and C.J. Buckley, eds., (Springer-Verlag, Berlin, 1992) p. 75.

16. B. Niemann, in X-Ray Microscopy III, A. G. Michette, G. R. Morrison, and C. J. Buckley, eds., Springer-Verlag (1990).

17. S. Williams, C. Jacobsen, S. Kirz, S. Lamm, and J. Van't Hof, ibid.

18. G. R. Morrison, et al., ibid.

19. M. Howells et al., Science, 238, 514 (1987).

20. I. McNulty et al., Science 256, 1009 (1992).

21. D. J. Thiel, D. H. Bilderback, A. Lewis, E. A. Stern, and T. Rich, preprint (submitted).

22. R. Bionta, E. Ables, O. Clamp, O. D. Edwards, P. C. Gabriele, K. Miller, L. L. Ott, K. M. Skullina, R. Tilley, T. Viada, Opt. Engrg., 576 (1990) and reference therein.

23. W. B. Yun, B. Lai, D. Legnini, Y. H. Xiao, d. Chrzas, K. M. Skulina, R. M. Bionta, V. White, F. Cerrina, SPIE Proc. 1740, 117 (1992), and reference therein.

24. Y. Wu, A. C. Thompson, J. H. Underwood, R.
D. Giauque, K. Chapman, M. L. Rivers, and K. W. Jones, Nucl. Instr. and Meth. A291, 146 (1990).

25. B. Lai, W. B. Yun, D. Legnini, Y. H. Xiao, J. Chrzas, "Hard X-ray Microimaging Techniques Based on Phase Zone Plates," SPIE Proc. 1741, 180 (1992).

26. A. C. Thompson, K.L. Chapman, G. E. Ice, C. J. Sparks, W. Yun, B. Lai, D. Legnini, P. J. Viccaro, and M. L. Rivers, Nucl. Instr. and Meth. A319, 320 (1992).

27. J. Kirz and D. Sayre, in Synchrotron Radiation Research (Plenum, New York, 1980) pp. 227-322.

28. G. Schmahl et al., in X-Ray Microscopy II, D. Sayre, M. Howells, J. Kirz and H. Rarback, eds., Springer-Verlag (1988).

29. B. N. Thomas, S. W. Barton, F. Novak, and S. A. Rice, J. Chem. Phys. 86, 1036 (1986).

30. D. K. Schwartz, A. Braslau, B. Ocko, and P. S. Pershan, Phys. Rev. A38, 5817 (1988).

31. H. You, C. A. Melendres, Z. Nagy, V. A. Maroni, W. Yun, and R. M. Yonco, Phys. Rev. B45, 11288 (1992).

32. J. M. Bloch, W. Yun, X. Yang, M. Ramanathan, P. A. Montano, and C. Capasso, Phys. Rev. Lett. 61, 2941 (1988),

33. W. C. Marra, P. H. Fuoss, and P. E. Eisenberger, Phys. Rev. Lett. 49, 1169 (1982).

34. M. F. Toney and B. M. Ocko, Synchrotron Radiation News 6, 28 (1993).

35. J. Zegenhagen and G. Materlik, Synchrotron Radiation News 6, 19 (1993).

36. M. Toney and Z. Rek, J. Mater. Res. 3, 351 (1988).

37. H. Guckel, Nucl. Instr. and Meth. B79, 247 (1993). 

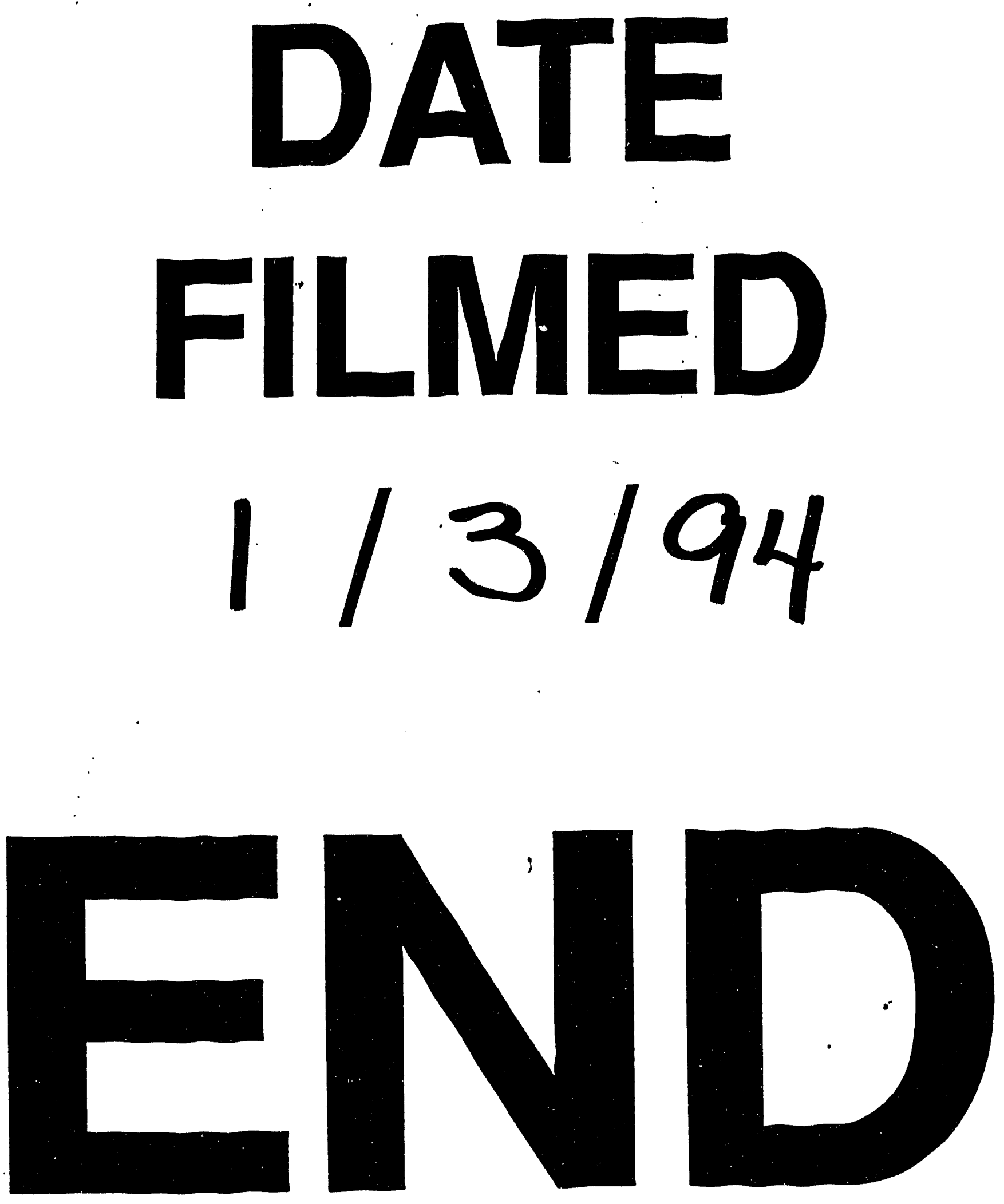
